\title{
Investigating the effect of eye cosmetics on the tear film: current insights
}

\section{Michael TM Wang \\ Jennifer P Craig}

Department of Ophthalmology, New Zealand National Eye Centre, The University of Auckland, Auckland, New Zealand
Correspondence: Jennifer P Craig

Department of Ophthalmology, New

Zealand National Eye Centre, The

University of Auckland

Private Bag 92019, Auckland II42, New

Zealand

Tel +6499238173

Fax +649367 7173

Email jp.craig@auckland.ac.nz
This article was published in the following Dove Press journal:

Clinical Optometry

\begin{abstract}
Eye cosmetics are frequently applied among female populations of all age groups around the world. However, the migration of cosmetic products across the eyelid margin has been reported, and this is thought to exacerbate tear film instability and symptoms of dry eye. Furthermore, numerous adverse effects and complications have also been reported with eye cosmetic wear, and the associated inflammatory responses may potentially increase the propensity toward ocular surface disease development. Prospective studies have demonstrated that eyeliner application at the inner eyelash line is associated with higher levels of tear film contamination and ocular discomfort than application at the outer periocular skin. A recent randomized trial also highlighted the potential for eye cosmetic wear to compromise the efficacy of lipid-based dry eye supplements. This review outlines the current evidence base and understanding regarding the periocular migration of eye cosmetic products, the effects of cosmetic product contamination on tear film function, and the use of dry eye treatments in eye cosmetic wearers.
\end{abstract}

Keywords: eyeliner, makeup, periocular, ocular surface, dry eye, contamination, cosmetic migration

\section{Introduction}

Eye cosmetics have been used since prehistoric times to emphasize and highlight the eyes (Figure 1). ${ }^{1,2}$ To this day, they remain widely used worldwide among female populations of all age groups. ${ }^{3,4}$ In addition to religious, cultural, and medicinal purposes, ${ }^{1,2}$ eye cosmetics are also commonly used to enhance perceived attractiveness and beauty. ${ }^{3-5}$ This is associated with profound psychosocial impacts, and many women report using cosmetic products to improve self-confidence., ${ }^{4,6}$ Market research from the United States and United Kingdom suggests that a large proportion of women use facial and eye cosmetics, ${ }^{4,6}$ with mascara, eyeliner, and eye shadow being among the most commonly applied products. ${ }^{4,6,7}$

Complications arising from the use of Kohl, a common eye cosmetic product in Indian and Arabic cultures, are well established, and include lead toxicity, conjunctival and periocular pigmentation..$^{8-10}$ In contrast, there has been little published literature on the long-term ocular effects of Western eye cosmetic formulations until recent decades, ${ }^{4}$ although the cosmetic industry is subjected to stringent regulation and rigorous safety assessments. ${ }^{4,6,7}$ Nevertheless, recent clinical evidence has demonstrated the migration of externally applied cosmetic material across the eyelid margin, ${ }^{11,12}$ and this is thought to predispose eye cosmetic wearers toward tear film instability and dry eye development. ${ }^{6,7,13-15}$

An understanding of the ocular effects of eye cosmetic wear and the efficacy of potential treatments is required to inform clinical advice and management. The purpose 

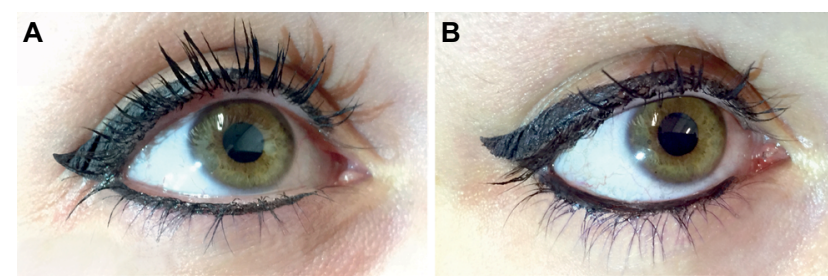

Figure I Eyeliner applied to $(\mathbf{A})$ the periocular skin of the lower eyelid, external to the lash line and (B) to the surface of the lower eyelid margin, within the lash line ("tightlining").

of this review was therefore to examine the current evidence base surrounding the ocular migration of eye cosmetic products, the effects of cosmetic product contamination on tear film function, and the use of dry eye treatments in eye cosmetic wearers.

\section{Ocular migration of eye cosmetic products}

The migration of externally applied eye cosmetics onto the ocular surface has been consistently reported. . $7,11,12,14^{2}$ There have been numerous anecdotal accounts of tear film contamination with eye cosmetic products during routine slit lamp examination. ${ }^{6} 12$ Prospective studies have confirmed that tear film contamination can result from passive and inadvertent migration of periocular cosmetic products across the eyelid margin, in addition to poor application techniques, direct accidental ocular instillation, and eye rubbing. ${ }^{6,11}$ Furthermore, this phenomenon is also thought to contribute toward some of the reported adverse effects associated with eye cosmetic wear, including posterior blepharitis, ocular surface irritation, tear film instability, conjunctival pigmentation, corneal epithelium inflammation, and keratitis., ${ }^{2,16-19}$

Eyeliner products are commonly applied to the periocular skin, immediately adjacent to, but external to the lash line (Figure 1A) or directly onto the lid margin at the mucocutaneous junction, also known as "tightlining" (Figure 1B). Clinical examples of periocular and inner lash line eyeliner application, highlighting the migration of cosmetic product onto the lid margin and onto the interpalpebral ocular surface are shown in Figure 2.

Two prospective studies have investigated the influence of the periocular location of cosmetic application on tear film contamination levels. ${ }^{11,12} \mathrm{~A}$ randomized parallel group study of 75 participants compared tear film contamination levels following application of a cosmetic product mixture of hydroxycellulose gel and fluorescein at the outer, mid, and inner eyelash lines. ${ }^{11}$ Ocular surface migration of the mixture was confirmed using the detection of fluorescence
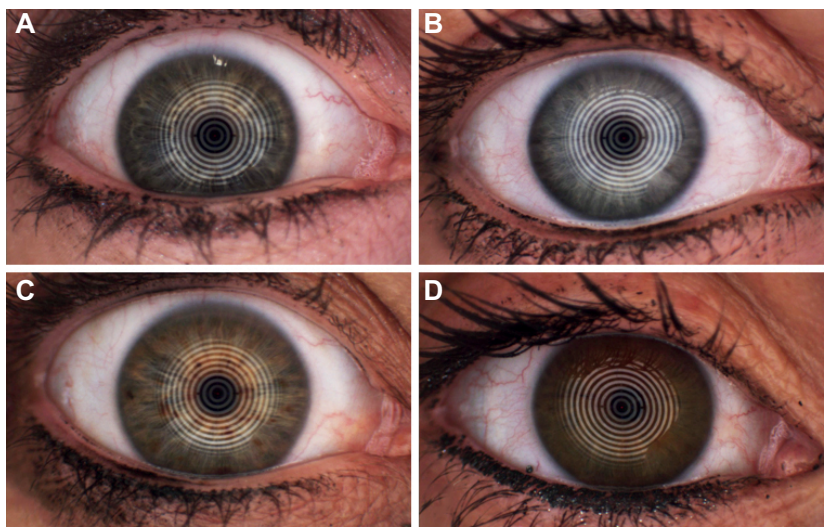

Figure 2 (A-C) Varying levels of eyeliner drift onto the eyelid margin following periocular eyeliner application, observed incidentally during bulbar hyperaemia examination; (D) Kohl eyeliner applied posterior to the lash line, directly onto the mucocutaneous junction, with visible debris within the interpalpebral zone.

under a slit lamp blue light filter. A Schirmer test paper strip was used for tear collection, and its color was used to semiquantitatively determine the level of tear film contamination. The results showed that ocular surface migration and tear film contamination levels were significantly greater and more rapid with product application at the inner eyelash line. In order to avoid brand bias and ocular irritation, the study investigators formulated their own cosmetic product mixture. However, the mixture was relatively more hydrophilic than some of the commercially available formulations, which potentially limits the applicability of the study findings to other cosmetic products.

Another pilot randomized crossover study investigated the migration of glitter particles into the tear film of three participants, following periocular skin and inner eyelash line application of a commercially available pencil eyeliner, consisting of a more hydrophobic mixture of waxes, oils, and pigments. ${ }^{12}$ High resolution slit lamp video recordings were performed to quantify the number of glitter particles suspended within the tear film. An example of pearlescent debris from cosmetics is shown in Figure 3. The study findings demonstrated that eyeliner application at the periocular skin was associated with slower and reduced levels of tear film contamination. Interestingly, tear film contamination levels generally peaked within 10 minutes of product application, and were negligible following 2 hours in both groups.

The exact mechanisms which facilitate the migration of externally applied cosmetic products across the eyelid margin are not fully understood. ${ }^{7,11,12,14}$ Nevertheless, a number of explanations for product migration have been proposed, with one such hypothesis suggesting that the eyelid movements during blinking may create mechanical forces which enhance 


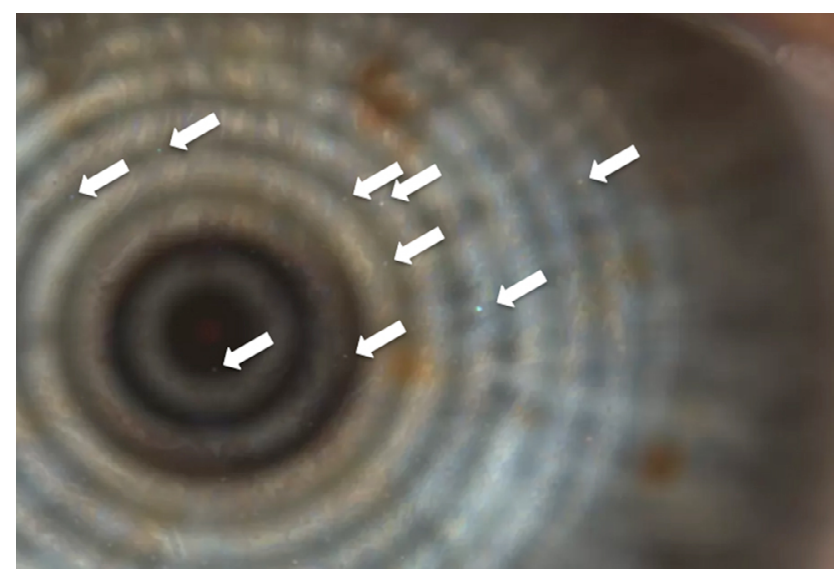

Figure 3 Pearlescent cosmetic particles in the tear film (arrows), visible incidentally during lipid evaluation (Oculus Keratograph 5M).

the movement of periocular particles toward the tear film, ${ }^{7,11,14}$ although the manner by which such mechanical forces are generated remains contentious. ${ }^{12}$ The recent identification of the "overblink" phenomenon ${ }^{20}$ would suggest that the direct apposition of the eyelid margins may not necessarily be implicated, while the role of mechanical rubbing remains unclear. ${ }^{12}$ In addition, forced blinking is thought to amplify the mechanisms that facilitate the transport of periocular particles, and may arise reflexively from ocular stimulation and irritation during cosmetic application or following initial product migration. ${ }^{11,14}$

Another hypothesis suggests that the action of the muscles of Riolan may also be involved in encouraging the migration of particles across the lid margin. ${ }^{12}$ The muscles of Riolan consist of a group of vertically aligned fibers which lie immediately subjacent to the periocular palpebral skin. ${ }^{21,22}$ Although the function of the muscles of Riolan is not fully understood, they are thought to be involved in promoting the expression of meibomian gland secretions, preserving the close approximation of the eyelid margin to the globe, and retaining the orientation of the eyelashes. ${ }^{12,21,22}$ In addition, they may also potentially effect minute vertical movements of the eyelid skin, which may further enhance the transfer of periocular particles toward the ocular surface. ${ }^{12,21}$ The relatively warmer temperature of the periocular eyelid skin relative to the external environment may reduce the viscosity of the eye cosmetic products applied, and further enhance motility. ${ }^{7}$ The suction forces that result from the surface tension of the tear meniscus may also promote the movement of cosmetic products that are in close proximity to the lid margin, toward the ocular surface. ${ }^{7,11}$

The movement of cosmetic material particles within the tear film itself is thought to be driven by a number of processes including diffusion, drift, and mass flow. ${ }^{7}$ The distribution of electrically neutral constituents may be dictated primarily by diffusional movement down the concentration gradient. Charged constituents can drift through the tear film according to the distribution of electrolytes and negatively charged glycocalyx molecules beneath the aqueous-mucin phase. The mass flow of the tear volume is driven by the lacrimal pump and blinking mechanism, facilitating the clearance of particulate matter within the tear film through the lacrimal drainage system.

\section{Effects on lipid layer quality}

The most superficial layer of the tear film is composed of a complex mixture of lipids secreted by the meibomian glands, ${ }^{23}$ and a continuous surface lipid layer is required to inhibit tear evaporation. ${ }^{24}$ It has been suggested that the migration of cosmetic products across the eyelid margin may potentially contribute toward an increase in debris within the surface tear film lipid layer, in meibomian gland blockage, and in meibum contamination. ${ }^{11,12,15}$ The resulting destabilization of the lipid layer can lead to increased tear evaporation and reduced tear film stability. ${ }^{6,714,24}$

An observational study of 180 participants reported a significant correlation between eye cosmetic wear and reduced tear film lipid layer thickness. ${ }^{25}$ The secondary analysis of another observational study of 281 participants found that female eye cosmetic wearers were less likely to exhibit foaming at the inner palpebral canthus than female participants who were not wearers of eye cosmetics. ${ }^{26}$ The eyelid margin foam was thought to be composed of sebum and meibum, and a reduction in foaming was found to be correlated with symptoms of dry eye. The study authors suggested that constituents of eye cosmetic product formulations may potentially bind with the amphipathic phospholipids, compromising the stability and preventing the formation of foam globules, which, in turn, could be related to general instability of the pre-ocular tear film. Interestingly, this interpretation differs significantly from the current understanding of eyelid margin foaming, which is thought to be associated with meibomian gland dysfunction and represent saponification of the lipid secretions induced by bacterial lipases. ${ }^{27}$ Furthermore, it is not known whether the confounding effects of regular eyelid hygiene regimens associated with eye cosmetic wear may have contributed toward the trends reported in the study.

A pilot infrared spectroscopy study was conducted to examine the effects of mixing liquid and pencil eyeliners on the molecular structure and lipid phase transition of human meibum. ${ }^{15}$ The study findings demonstrated that the 
cosmetic products tested exhibited considerably higher lipid phase transition temperatures than meibum. The lipid phase transition temperature of human meibum was also found to increase by $4.2^{\circ} \mathrm{C}$ when mixed with the pencil eyeliner product, and the minimum frequency, enthalpy and entropy of phase transition decreased significantly. Overall, the changes in lipid order observed following the application of pencil eyeliner to human meibum represented an increase in viscosity, which was thought to have the potential of exerting adverse effects on tear film stability.

A randomized crossover study of 24 participants compared the effects of 7-day pencil eyeliner application at the periorbital skin and mucocutaneous junction. ${ }^{28}$ Interestingly, the study found that mucocutaneous junction application was associated with improved lipid layer thickness, decreased ocular comfort, and no changes in tear film stability. Furthermore, the Ocular Surface Disease Index (OSDI) scores were significantly poorer following eyeliner application at the mucocutaneous junction than the periorbital skin. The study investigators concluded that the migration of lipophilic eyeliner constituents was likely to explain the increased lipid content observed within the tear film. However, the adverse impacts on symptomology suggested that ocular surface homeostasis had been disrupted by the migration of cosmetic products.

The potential role of preservatives used within eye cosmetic formulations has also been raised. A review suggested that benzylalkonium chloride (BAK), a quaternary ammonium preservative, may contribute toward lipid layer destabilization through its detergent-like tensioactive effects. ${ }^{7}$ However, it is noted that BAK is no longer widely used in eye cosmetic products, partly due to its reduced preservative efficacy within formulations containing solid particulate matter or ionic emulsifications. ${ }^{29}$ Nevertheless, the review also proposed a model by which lipophilic constituents of cosmetic formulations may disrupt the integrity of the tear film lipid layer. ${ }^{7}$ The model suggests that the lipophilic substances may initially diffuse through the surface lipid layer. However, their inability to dissolve within the aqueous phase of the tear film could lead to their accumulation at the lipid-aqueous interface, compromising the stability of the overlying tear film lipid layer.

The adverse effects of eye cosmetic removal products on tear film parameters have also been reported. An in vivo clinical study demonstrated the migration of cosmetic removal products into the tear film, following application of the solution over closed eyelids. ${ }^{30}$ A prospective study of 20 participants compared the effects of three eye cosmetic removal products on tear film measurements, including an oil- and alcohol-free formulation, an oil-based microemulsion, and a hyaluronic acid-containing micelle solution. ${ }^{31}$ The results showed that all three products effected an increase in tear film evaporation, and, rather unexpectedly, the oil-based microemulsion was associated with the greatest reduction in lipid layer quality and tear film stability.

Nevertheless, despite the unfavorable impact of the migration of cosmetic products and removers across the eyelid margin, it has been suggested that the digital manipulation and eyelid hygiene regimens associated with regular removal of eye cosmetic products might introduce somewhat paradoxical confounding effects. ${ }^{4}$ Eyelid hygiene and digital expression of meibomian glands are both commonly recommended components of the multi-modal management of meibomian gland dysfunction, the leading cause of evaporative dry eye. ${ }^{32,33}$ Digital expression of meibomian glands has been shown to increase tear film stability and reduce the rate of tear evaporation. ${ }^{34,35}$ It is not known whether regular eyelid hygiene and digital manipulation associated with routine removal of eye cosmetic products may potentially counteract some of the adverse effects associated with cosmetic product migration into the tear film. ${ }^{4}$

\section{Ocular surface inflammatory effects}

Numerous adverse effects and complications have been reported in association with eye cosmetic wear. ${ }^{2,6,7}$ There is an increased risk of ocular infection with microbial contamination of the products, which may arise from multiple sources, such as during the manufacturing process, reduced preservative efficacy with the age of the product, and shared use of cosmetic products amongst multiple users. ${ }^{36-39}$ Ocular Demodex mites have a high affinity toward oil-rich environments, such as the meibomian glands, and may also survive in oil-based cosmetic products and applicators. ${ }^{6}$ Corneal trauma is a recognized potential complication from the use of mascara applicator wands (Figure 4). ${ }^{40-42}$ Eyelid dermatitis and ocular surface irritation can also occur in response to several constituents within eye cosmetic formulations including fragrances, preservatives, antioxidants, emollients, resins, nickel-containing pigments, and pearlescent additives. ${ }^{43,44} \mathrm{In}$ addition, posterior blepharitis, ocular surface irritation, conjunctival pigmentation, and keratitis have also been reported in association with eye cosmetic wear. ${ }^{2,7,16-19}$

The adverse effects reported would suggest that the constituents of eye cosmetic formulations may have the potential to trigger ocular surface inflammatory pathways. This can serve as an additional entry point to the vicious circle of dry 


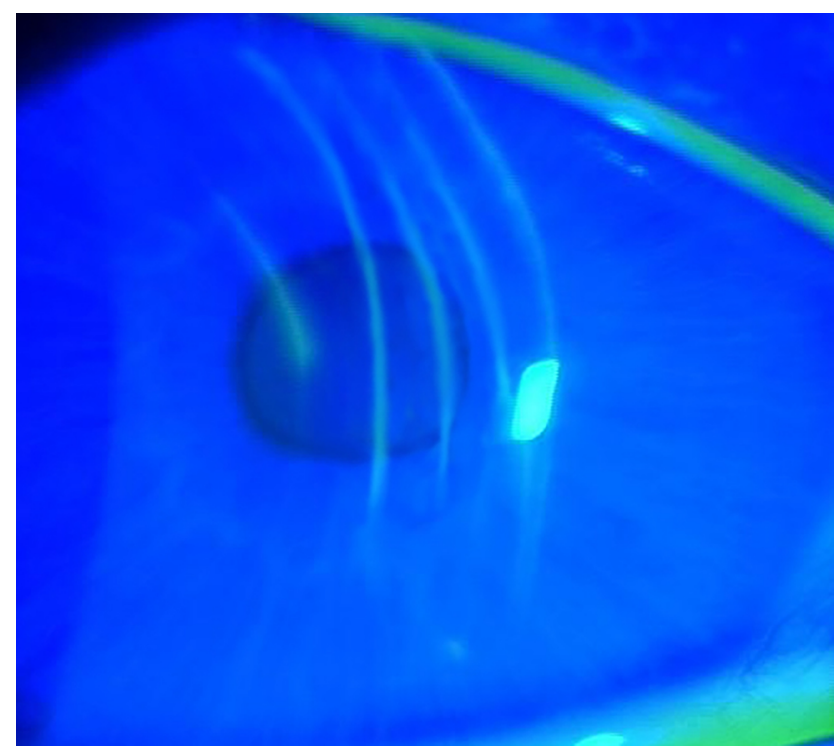

Figure 4 Corneal abrasions induced by a mascara wand, observed under blue ligh following sodium fluorescein instillation.

eye disease ${ }^{45}$ independent of the tear film hyper-evaporative mechanisms driven by the compromised surface lipid layer quality. Inflammatory mediators within the tear film can lead to ocular surface epithelial damage, goblet cell loss, and disturbances in glycocalyx mucin expression. These inflammatory changes can exacerbate any pre-existing susceptibility to tear film instability and hyperosmolarity. The resulting ocular surface desiccation, frictional damage, and inflammatory cascades may also adversely affect aqueous tear production, which would act synergistically with the hyper-evaporative mechanisms driven by tear film instability to further perpetuate the vicious circle of dry eye disease.

It has also been suggested that the potential influx and accumulation of hydrophilic constituents of cosmetic formulations within the aqueous-mucin phase of the tear film may directly increase tear film osmolarity. ${ }^{7}$ Tear film hyperosmolarity can lead to epithelial damage, both directly and indirectly, through mediating various inflammatory pathways. The resulting ocular surface changes can predispose toward poorer tear film stability. Furthermore, the movement of particulate matter within the aqueous-mucin phase may also affect the viscoelasticity of the tear film, which can also contribute toward tear film instability.

\section{Effects on ocular comfort}

The adverse impact of eye cosmetic products on tear film stability would suggest that their use is associated with poorer ocular comfort and dry eye symptomology, ${ }^{11,12,14,28}$ although, it has been acknowledged that eyelid hygiene regimens and digital manipulation associated with routine removal of cosmetic products may potentially introduce confounding effects. ${ }^{4}$

A cross-sectional survey of 1360 female respondents was conducted to explore the relationship between eye cosmetic usage and ocular comfort. ${ }^{4}$ The survey results showed that eye cosmetic wearers displayed marginally poorer OSDI scores than participants who did not use eye cosmetics, although the trends did not reach statistical significance. Among eye cosmetic wearers, perceived ocular comfort was significantly poorer during days when make-up products were applied. The frequency and specific product applied were found not to be correlated with OSDI scores. Nevertheless, the potential for self-selection bias cannot be excluded, whereby respondents susceptible to symptoms of dry eye may potentially limit their pattern of eye cosmetic wear, in order to minimize ocular discomfort.

A randomized crossover study of 20 female participants showed that perceived ocular comfort decreased following 7-day pencil eyeliner application to either the periocular skin and mucocutaneous junction. ${ }^{28}$ Furthermore, mucocutaneous junction application was associated with poorer OSDI scores than periocular skin application, and the study investigators attributed this trend toward the greater levels of tear film contamination when the product was applied closer to the eye's surface.

Another prospective study involving 410 participants tracked subjective and objective measurements of ocular irritation during a 2-hour period following provocative instillation of neat formulations of mascara, powder eye shadow, eye cosmetic remover, and liquid cosmetics into the inferior fornix. ${ }^{16}$ The study intended to mimic accidental ocular instillation of cosmetic products, and found that powder eye shadow was associated with the most severe sodium fluorescein staining scores. The results also demonstrated that the subjective irritation scores generally peaked within 30 seconds following ocular instillation of cosmetic products, and symptoms usually resolved within 15 minutes.

\section{Dry eye treatments in eye cosmetic wearers}

Dry eye disease is one of the most commonly encountered ophthalmic conditions in clinical practice, and is recognized to have significant effects on vision, ocular comfort, and quality of life. ${ }^{46}$ Epidemiological studies have consistently reported a higher prevalence of dry eye among females. ${ }^{47}$ Furthermore, there is thought to be an increased propensity for eye cosmetic wearers to develop symptoms of dry eye, due to the reduced tear film stability and lipid layer quality 
associated with the migration of periocular cosmetic products across the eyelid margin. ${ }^{6,7,13-15}$ With the higher prevalence of dry eye disease and widespread use of eye cosmetics among the female population, research investigating the compatibility and efficacy of dry eye treatments in patients concurrently wearing eye cosmetics is required to inform clinical advice and management.

A prospective study of 75 participants demonstrated that saline eye drop instillation exacerbated the migration of a hydrophilic periocular mixture of hydroxyethyl cellulose gel and aqueous sodium fluorescein into the tear film. ${ }^{11} \mathrm{~A}$ more recent investigator-masked randomized paired-eye trial of 50 participants was conducted to compare the effects of a lipidcontaining lubricant eye drop and phospholipid liposomal spray on the tear film function of eye cosmetic wearers. ${ }^{14} \mathrm{~A}$ pencil eyeliner was applied to the periocular skin anterior to the lash line 15 minutes prior to treatment application. The study results showed that neither treatment adversely affected periocular appearance, as might occur if displacement or smudging of the product occurred, although particulate tear film contamination was observed on slit lamp examination in a greater proportion of eyes following application of either treatment. The results of the two studies suggest that eye drop instillation and liposomal spray application may both be associated with increased tear film contamination in eye cosmetic wearers. The corneal sensation elicited by eye drop instillation can reflexively induce forceful and excessive blinking, which may promote increased migration of cosmetic products. ${ }^{11,14}$ Furthermore, the overflow of fluid following eye drop instillation may allow the suction forces created by the surface tension of the tear film and eye drop solution to act over a greater surface area, drawing in an increased amount of cosmetic material. ${ }^{7,11}$ The migration of liposomes delivered by the phospholipid spray across the eyelid margin has previously been demonstrated, ${ }^{48-50}$ and the interaction between the liposomes and cosmetic products may potentially contribute toward the greater levels of tear film contamination observed. ${ }^{14}$

Interestingly, in the second study, no significant changes were detected in tear film stability or evaporation, despite improvements in tear film lipid layer thickness being observed following both treatments. ${ }^{14}$ These trends contrast greatly with earlier studies which demonstrate the clinical efficacy of both dry eye treatments in improving tear film stability in participants who were not wearing eye cosmetics. ${ }^{48,51}$ Although the lipid supplementation provided by both the lubricant eye drop and liposomal spray effected an increase in lipid layer thickness, these effects may potentially have been negated by the destabilizing action associated with the increased migration of cosmetic products into the tear film. ${ }^{14}$ This suggests that the ability to achieve the full clinical potential of either dry eye treatment might be compromised in eye cosmetic wearers. Whether this is a transient or persistent effect is unknown, and future long-term studies are required to assess potential cumulative and delayed effects of dry eye treatments in wearers of eye cosmetics.

\section{Conclusion}

Eye cosmetics are used extensively worldwide, predominantly by female populations, and across almost all age groups. Prospective studies have confirmed that tear film contamination can result from the passive migration of both hydrophilic and hydrophobic cosmetic formulations toward the ocular surface. Experimental and clinical evidence would suggest that the migration of such products across the eyelid margin may destabilize the surface tear film lipid layer, thereby predisposing wearers toward poorer tear film stability and evaporative dry eye symptoms. However, mixed findings have been reported in clinical studies, perhaps as a result of confounding factors that cannot be excluded, such as regular digital manipulation and eyelid hygiene regimens associated with eye cosmetic removal routines.

In addition to lipid layer destabilization, a number of other ocular surface adverse effects and complications have been reported in association with eye cosmetic wear. This suggests the potential presence of pro-inflammatory agents within cosmetic formulations, which can increase the propensity toward ocular surface disease development.

Interestingly, the majority of published clinical studies have focused on the effects of eyeliners, and future long-term prospective research is required to establish the effects of a broader range of cosmetic products on tear film function, including those of facial creams and cleansers. Nevertheless, randomized studies have shown that eyeliner application at the inner eyelash line is associated with greater levels of tear film contamination and ocular discomfort than the external periocular skin. The potential for supplemental adverse impacts from eyeliner application directly over the meibomian gland orifices in the longer term also needs to be considered in future studies, but based on current evidence, eye cosmetic wearers should be advised to avoid product application to the inner eyelash line, in order to minimize cosmetic product migration across the eyelid margin.

The higher prevalence of dry eye disease among females and the widespread use of eye cosmetics necessitate research assessing the clinical efficacy of dry eye treatments in eye 
cosmetic wearers. A recent randomized trial showed that both lipid-containing eye drops and liposomal sprays appear to exacerbate the migration of cosmetic products across the eyelid margin. Although significant increases in lipid layer thickness were observed following both treatments, their clinical efficacy in improving tear film stability appeared to be compromised. Future long-term trials understanding the effects of cosmetic use and exploring the efficacy and compatibility of a wider range of dry eye treatments in eye cosmetic wearers are required to inform clinical management.

\section{Acknowledgment}

This research did not receive any specific grant from funding agencies in the public, commercial, or not-for-profit sectors.

\section{Disclosure}

JPC has previously received research funding or materials, unrelated to the content of this manuscript, from: Oculeve: Allergan, Manuka Health NZ, E-Swin, CooperVision, Alcon, Optima Pharmaceuticals, Akorn, Medmont, and serves as a consultant to Carl Zeiss Meditec and Azura Ophthalmics. MTMW reports no conflicts of interest in this work.

\section{References}

1. Murube J. Ocular cosmetics in ancient times. Ocul Surf. 2013;11(1):2-7.

2. Coroneo MT, Rosenberg ML, Cheung LM. Ocular effects of cosmetic products and procedures. Ocul Surf. 2006;4(2):94-102.

3. Murube J. Ocular cosmetics in modern times. Ocul Surf. 2013; 11(2):60-64

4. Ng A, Evans K, North R, Purslow C. Eye cosmetic usage and associated ocular comfort. Ophthalmic Physiol Opt. 2012;32(6):501-507.

5. Mulhern R, Fieldman G, Hussey T, Lévêque JL, Pineau P. Do cosmetics enhance female Caucasian facial attractiveness? Int J Cosmet Sci. 2003;25(4):199-205.

6. Ng A, Evans K, North RV, Jones L, Purslow C. Impact of eye cosmetics on the eye, adnexa, and ocular surface. Eye Contact Lens. 2016;42(4): 211-220.

7. Malik A, Claoué C. Transport and interaction of cosmetic product material within the ocular surface: beauty and the beastly symptoms of toxic tears. Cont Lens Anterior Eye. 2012;35(6):247-259.

8. El Safoury OS, El Fatah DS, Ibrahim M. Treatment of periocular hyperpigmentation due to lead of kohl (surma) by penicillamine: a single group non-randomized clinical trial. Indian J Dermatol. 2009;54(4):361-363.

9. Al-Ashban RM, Aslam M, Shah AH. Kohl (surma): a toxic traditional eye cosmetic study in Saudi Arabia. Public Health. 2004;118(4):292-298.

10. Hidayat AA, Weatherhead RG, al-Rajhi A, Johnson FB. Conjunctival and lacrimal sac pigmentation by kohl (eyeliner). $\mathrm{Br} J$ Ophthalmol. 1997;81(5):418.

11. Goto T, Zheng X, Gibbon L, Ohashi Y. Cosmetic product migration onto the ocular surface: exacerbation of migration after eyedrop instillation. Cornea. 2010;29(4):400-403.

12. $\mathrm{Ng} \mathrm{A}$, Evans K, North RV, Purslow C. Migration of cosmetic products into the tear film. Eye Contact Lens. 2015;41(5):304-309.

13. Lozato PA, Pisella PJ, Baudouin C. Phase lipidique du film lacrymal physiologie et pathologie. [The lipid layer of the lacrimal tear film: physiology and pathology]. J Fr Ophthalmol. 2001;24(6):643-658.French.
14. Wang MTM, Cho ISH, Jung SH, Craig JP. Effect of lipid-based dry eye supplements on the tear film in wearers of eye cosmetics. Cont Lens Anterior Eye. 2017;40(4):236-241.

15. Hunter M, Bhola R, Yappert MC, Borchman D, Gerlach D. Pilot study of the influence of eyeliner cosmetics on the molecular structure of human meibum. Ophthalmic Res. 2015;53(3):131-135.

16. Gao Y, Kanengiser BE. Categorical evaluation of the ocular irritancy of cosmetic and consumer products by human ocular instillation procedures. J Cosmet Sci. 2004;55(4):317-325.

17. Platia EV, Michels RG, Green WR. Eye-cosmetic-induced conjunctival pigmentation. Ann Ophthalmol. 1978;10(4):501-504.

18. Mselle J. The role of eyelash dyes in allergic eye diseases. Trop Doct. 2004;34(4):235-236.

19. Kaiserman I. Severe allergic blepharoconjunctivitis induced by a dye for eyelashes and eyebrows. Ocul Immunol Inflamm. 2003;11(2):149-151.

20. Pult H, Riede-Pult BH, Murphy PJ. A new perspective on spontaneous blinks. Ophthalmol. 2013;120(5):1086-1091.

21. Lipham WJ, Tawfik HA, Dutton JJ. A histologic analysis and threedimensional reconstruction of the muscle of Riolan. Ophthal Plastic Reconstr Surg. 2002;18(2):93-98.

22. Kakizaki H, Nakano T, Ikeda H, Selva D, Leibovitch I. Tarsal elastic fiber distribution: an anatomic study. Ophthal Plastic Reconstr Surg. 2011;27(2):128-129.

23. Willcox MDP, Argüeso P, Georgiev GA, et al. TFOS DEWS II Tear Film Report. Ocul Surf. 2017;15(3):366-403.

24. Craig JP, Tomlinson A. Importance of the lipid layer in human tear film stability and evaporation. Optom Vis Sci. 1997;74(1):8-13.

25. Franck C. Fatty layer of the precorneal film in the 'office eye syndrome'. Acta Ophthalmol. 1991;69(6):737-743.

26. Franck C, Skov P. Foam at inner eye canthus in office workers, compared with an average Danish population as control group. Acta Ophthalmol. 1989;67(1):61-68.

27. Siddireddy JS, Vijay AK, Tan J, Willcox M. The eyelids and tear film in contact lens discomfort. Cont Lens Anterior Eye. Oct 182017 [Epub ahead of print].

28. Ng A, Evans K, North R, Purslow C. The effects of cosmetic eye pencil application on the tear film and ocular surface. Invest Ophthalmol Vis Sci. 2013;54(15):952.

29. Geis PA, Steinberg D. Response to: Malik and Claoue - transport and interaction of cosmetic product material within the ocular surface: beauty and the beastly symptoms of toxic tears. Cont Lens Anterior Eye. 2013;36(3):151.

30. Debbasch C, Ebenhahn C, Dami N, et al. Eye irritation of low-irritant cosmetic formulations: correlation of in vitro results with clinical data and product composition. Food Chem Toxicol. 2005;43(1):155-165.

31. Pearce EI, Harvey-Brown M, Higginson C. The interaction between eye make-up removers and the tear film. Poster presented at: 6th International Conference on the Tear Film and Ocular Surface; September 22-25, 2010; Florence, Italy.

32. Jones L, Downie LE, Korb D, et al. TFOS DEWS II Management and Therapy Report. Ocul Surf. 2017;15(3):575-628.

33. Geerling G, Tauber J, Baudouin C, et al. The International Workshop on Meibomian Gland Dysfunction: Report of the Subcommittee on Management and Treatment of Meibomian Gland Dysfunction. Invest Ophthalmol Vis Sci. 2011;52(4):2050-2064.

34. Craig JP, Blades K, Patel S. Tear lipid layer structure and stability following expression of the meibomian glands. Ophthalmic Physiol Opt. 1995;15(6):569-574.

35. Arciniega JC, Wojtowicz JC, Mohamed EM, McCulley JP. Changes in the evaporation rate of tear film after digital expression of meibomian glands in patients with and without dry eye. Cornea. 2011;30(8):843-847.

36. Lundov MD, Zachariae C. Recalls of microbiologically contaminated cosmetics in EU from 2005 to May 2008. Int J Cosmet Sci. 2008;30(6): 471-474.

37. Pack LD, Wickham MG, Enloe RA, Hill DN. Microbial contamination associated with mascara use. Optometry. 2008;79(10):587-593. 
38. Wilson LA, Julian AJ, Ahearn DG. The survival and growth of microorganisms in mascara during use. Am J Ophthalmol. 1975;79(4): 596-601.

39. Wilson LA, Kuehne JW, Hall SW, Ahearn DG. Microbial contamination in ocular cosmetics. Am J Ophthalmol. 1971;71(6):1298-1302.

40. Wilson SE, Bannan RA, McDonald MB, Kaufman HE. Corneal trauma and infection caused by manipulation of the eyelashes after application of mascara. Cornea. 1990;9(2):181-182.

41. Reid FR, Wood TO. Pseudomonas corneal ulcer. The causative role of contaminated eye cosmetics. Arch Ophthalmol. 1979;97(9):1640-1641.

42. Wilson LA, Ahearn DG. Pseudomonas-induced corneal ulcers associated with contaminated eye mascaras. Am JOphthalmol. 1977;84(1):112-119.

43. Draelos ZD. Special considerations in eye cosmetics. Clin Dermatol. 2001;19(4):424-430.

44. Adams RM, Maibach HI. A five-year study of cosmetic reactions. J Am Acad Dermatol. 1985;13(6):1062-1069.

45. Bron AJ, de Paiva CS, Chauhan SK, et al. TFOS DEWS II Pathophysiology Report. Ocul Surf. 2017;15(3):438-510.
46. Craig JP, Nelson JD, Azar DT, et al. TFOS DEWS II Report Executive Summary. Ocul Surf. 2017;15(4):802-812.

47. Stapleton F, Alves M, Bunya VY, et al. TFOS DEWS II Epidemiology Report. Ocul Surf. 2017;15(3):334-365.

48. Craig JP, Purslow C, Murphy PJ, Wolffsohn JS. Effect of a liposomal spray on the pre-ocular tear film. Cont Lens Anterior Eye. 2010;33(2): 83-87.

49. Wang MT, Ganesalingam K, Loh CS, et al. Compatibility of phospholipid liposomal spray with silicone hydrogel contact lens wear. Cont Lens Anterior Eye. 2017;40(1):53-58.

50. Lee S, Dausch S, Maierhofer G, Dausch D. Ein neues Therapiekonzept zur Behandlung des Trockenen Auges - die Verwendung von Phospholipid-Liposomen. [A new therapy concept for the treatment of dry eye - the usefulness of phospholipid liposomes]. Klin Monbl Augenheilkd. 2004;221(10):825-836. German.

51. Gokul A, Wang MTM, Craig JP. Tear lipid supplement prophylaxis against dry eye in adverse environments. Cont Lens Anterior Eye. 2018;41(1):97-100.
Clinical Optometry

\section{Publish your work in this journal}

Clinical Optometry is an international, peer-reviewed, open access journa publishing original research, basic science, clinical and epidemiologica studies, reviews and evaluations on clinical optometry. All aspects of patient care are addressed within the journal as well as the practice of optometry including economic and business analyses. Basic and clinical

Submit your manuscript here: https://www.dovepress.com/clinical-optometry-journal
Dovepress

research papers are published that cover all aspects of optics, refraction and its application to the theory and practice of optometry. The manuscript management system is completely online and includes a very quick and fair peer-review system, which is all easy to use. Visit http://www.dovepress. com/testimonials.php to read real quotes from published authors. 